\title{
Call for Evaluation and Reporting of the Equity Impact of Culturally Responsive Nutrition Interventions
}

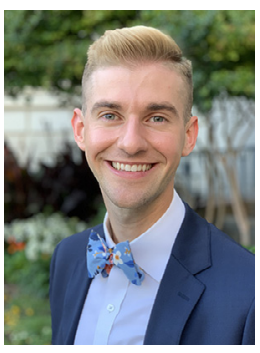

Landry

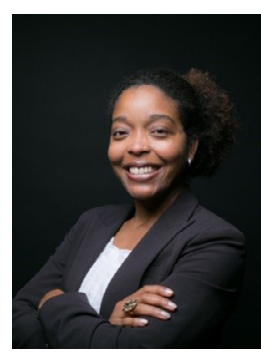

Alford

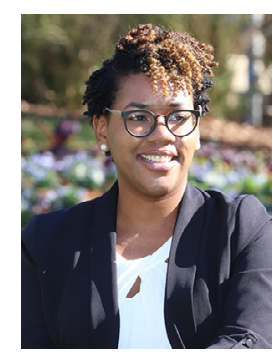

Singleton
We believe nutrition educators and researchers recognize the importance of health equity but do not have a clear sense of how to quantify, and ultimately, achieve equity. A 2014 study con-

In the United States, socially and economically disadvantaged populations (e.g., people of color, immigrants, indigenous people, the homeless, LGBTQ+ individuals) face unique nutritional challenges that increase their risk of adverse health outcomes. ${ }^{1}$ Unfortunately, many of these challenges are driven by systemic oppression and various structural inequities, which have hindered, and in many cases, completely prohibited access to opportunities for maintaining a healthy diet and lifestyle. ${ }^{2}$

Public health professionals and nutrition educators have long acknowledged the need to address the structural inequalities that contribute to poor nutrition among disadvantaged populations. In response, the field has introduced several culturally responsive nutrition interventions in recent years that specifically emphasized the importance of health equity. ${ }^{3}$ Experts designed these interventions to be culturally responsive by engaging target populations and being inclusive of local cultural norms, food preferences, and socioenvironmental landscapes. Furthermore, they emphasized the importance of health equity by improving relationships, resources, policies, systems, and environments in an equitable and sustainable manner. ${ }^{4}$ Nevertheless, despite this increase in culturally responsive nutrition interventions, we know very little about the impact they have had on health equity. zation's Commission on Social Determinants of Health revealed that most health impact assessments do not adequately examine health equity. ${ }^{5}$ Reasons for this include the absence of definitions, poor data, a lack of methods and measurement tools, and practitioner inability to evaluate values like social justice. As a field, we are quite capable of measuring and reporting on nutrition and health-related outcomes (e.g., dietary intake, obesity status). However, evaluation plans for interventions often do not include a component that measures the health equity impact of the intervention. In our experience, if an intervention's evaluation plan does include this component, the findings are often not published or readily available to the public.

To improve the field's understanding of the equity impact of culturally responsive nutrition interventions, we believe nutrition educators and researchers must expand their evaluation efforts to study the effects of their interventions on health equity. Furthermore, they must report these findings to the field via peer-reviewed publications or public reports. This calls for (1) the revisiting of existing definitions, frameworks and resources, (2) the development of new data collection tools and measurable outcomes, (3) the incorporation of implementation science methodologies $^{6}$ capable of assessing the fidelity ducted by the World Health Organi- of the health equity dimension of interventions, and (4) greater emphasis on health equity evaluation and reporting among researchers and funding agencies.

The field has taken some positive steps in the right direction by introducing new health equity frameworks capable of guiding this work. 6,7 Policy, systems, and environmental (PSE) change approaches are key to addressing health inequities. ${ }^{4}$ In 2019, Dr. Shiriki Kumanyika published a framework for increasing the equity impact in obesity prevention interventions in the American Journal of Public Health. ${ }^{7}$ This framework offers an in-depth perspective on addressing equity barriers that impede the effectiveness of PSE obesity interventions. This framework advocates for increasing healthy options, reducing deterrents, improving social and economic resources, and building community capacity. A next logical step might be the development of measures capable of capturing these constructs and the creation of evaluation protocols that encompass these equity-related outcomes and goals. Additionally, researchers can use the CONSORT-Equity 2017 (Consolidated Standards of Reporting Trials) statement, an extension of existing trial CONSORT reporting guidance, to improve the collection and dissemination of health equity data in randomized trials.

In summary, nutrition educators and researchers should systematically evaluate and report the equity impact of nutrition interventions to advance the field's understanding of health inequity. The field has emphasized cultural responsiveness and inclusion in recent years, but still has little understanding of whether nutrition interventions are truly addressing the root causes of inequities. With social consciousness about health equity increasing in the United States, it is more important 
than ever to improve our understanding of the equity impact of culturally responsive nutrition interventions. We hope this call leads to a permanent paradigm shift in how nutrition educators and researchers evaluate and report intervention findings.

Matthew J. Landry, PhD, RDN, LDN Stanford Prevention Research Center, School of Medicine, Stanford University, Palo Alto, CA

Chair-Elect, Public Health Nutrition Division of the Society for Nutrition Education and Behavior Shana Alford, MPP, BBA Common Threads, Chicago, IL Public Health Nutrition Division of the Society for Nutrition Education and Behavior

Chelsea R. Singleton, PhD, MPH Department of Social, Behavioral, and Population Sciences, Tulane School of
Public Health and Tropical Medicine, New Orleans, LA

Chair, Public Health Nutrition Division of the Society for Nutrition Education and Behavior

\section{REFERENCES}

1. US Department of Health and Human Services. Disparities. Healthypeople.gov. https://www.healthypeople.gov/2020/ about/foundation-health-measures/ Disparities. Accessed December 21, 2021.

2. Taylor MM. The argument for social justice. In: The Obesity Epidemic: Why a Social Justice Perspective Matters. Macmillan; 2018.

3. Bailey ZD, Krieger N, Agénor $M$, Graves J, Linos N, Bassett MT. Structural racism and health inequities in the USA: evidence and interventions. Lancet. 2017;389:1453-1463.
4. Story MT, Duffy E. Supporting healthy eating: synergistic effects of nutrition education paired with policy, systems, and environmental changes. In: Black MM, Delichatsios HK, Story MT, eds. Nutrition Education: Strategies for Improving Nutrition and Healthy Eating in Individuals and Communities, Nestle Nutrition Institute; 2020:69-81.

5. Snell-Rood C, Jaramillo ET, Hamilton AB, Raskin SE, Nicosia FM, Willging C. Advancing health equity through a theoretically critical implementation science. Transl Behav Med. 2021;11:1617-1625. https://doi.org/10.1093/tbm/ibab008.

6. Kumanyika SK. A framework for increasing equity impact in obesity prevention. Am J Public Health. 2019;109:1350-1357.

7. Welch VA, Norheim OF, Jull J, Cookson R, Sommerfelt H, Tugwell P. CONSORT-Equity 2017 extension and elaboration for better reporting of health equity in randomised trials. BMJ. 2017;359:j5085. https://doi.org/10.1136/bmj.j5085. 